$B N L--48720$

DE93 010870

\title{
The Effect of Synchrobetatron Coupling on the Dynamic Aperture
}

\author{
G. Parzen
}

\section{March 1993}

\section{DISCLAIMER}

This report was prepared as an account of work sponsored by an agency of the United States Government. Neither the United Siates Government nor any agency thereof, nor any of their employees, makes any warranty, express or implied, or assumes any legal liability or responsibility for the accuracy, completeness, or usefulness of any information, apparatus, product, or process disclosed, or represents that its use would not infringe privately owned rights. Reference herein to any specific commercial product, process, or service by trade name, trademark, manufacturer, or otherwise does not necessarily constitute or imply its endorsement, recommendation, or favoring by the United States Government or any agency thereof. The views and opinions of authors expressed herein do not necessarily state or reflect those of the United States Government or any agency thereof.

\author{
Broolhaven National Laboratory \\ Associated Universities, Inc. \\ Upton, NY 11973
}




\title{
THE EFFECT OF SYNCHROBETATRON COUPLING ON THE DYNAMIC
}

\section{APERTURE}

\author{
G. Parzen \\ Brookhaven National Laboratory \\ Upton, NY 11973, USA
}

\section{INTRODUCTION}

The effect of synchrobetatron coupling on the dynamic aperture was studied by comparing the dynamic aperture for a particle with a large fixed $\Delta p / p$, no synchrotron oscillations present, with the dynamic aperture for a particle with a synchrotron oscillation amplitude of the same $\Delta p / p$. The particle with the synchrotron oscillation present was found to have a smaller dynamic aperture than that of the particle with the fixed $\Delta p / p$. It is suggested that this reduction in dynamic aperture may be due to a non- linear coupling between the longitudinal and transverse motions.

For RHIC, whose lattice and RF were used in this study, the longitudinal phase space is much larger than the transverse phase space by a factor of several thousand, and a small amount of coupling can cause considerable growth in the transverse motion.

The effect is most pronounced at lower energies in RHIC, where larger momentum spread and transverse amplitudes are required. At $\gamma=30$ in RHIC, with a synchrotron oscillation amplitude of $\Delta p / p=0.005$, the dynamic aperture is reduced by about $6 \mathrm{~mm}$ by the presence of the synchrotron oscillations. The effect may be more important for RHIC than for other superconducting proton colliders, because of its relatively low energy and because of the importance of intrabeam scattering for heavy ions. This results in larger dynamic aperture requirements for RHIC both in transverse space and in momentum spread.

\section{TRACKING RESULTS}

The largest effect is seen at $\gamma=30$ for RHIC, where the beam momentum spread can grow to $\Delta p / p= \pm 0.005$ after 10 hours. Results are shown in Fig. 1 for the dynamic aperture as a function of the size of the synchrotron oscillation amplitude, $\Delta p / p$. The dynamic aperture drops from $A_{S L}=14.5$

* Work performed under the auspices of the U.S. Department of Energy. 
$\mathrm{mm}$ at $\Delta p / p=0$ to $A_{S L}=8.5 \mathrm{~mm}$ at $\Delta p / p=0.005$. Also shown in Fig. 1 is the dynamic aperture found when the particle momentum is held fixed at some level, and no synchrotron oscillations are present. This curve is relatively flat and at $\Delta p / p=0.005$, there is a difference of $6 \mathrm{~mm}$ between the two curves.

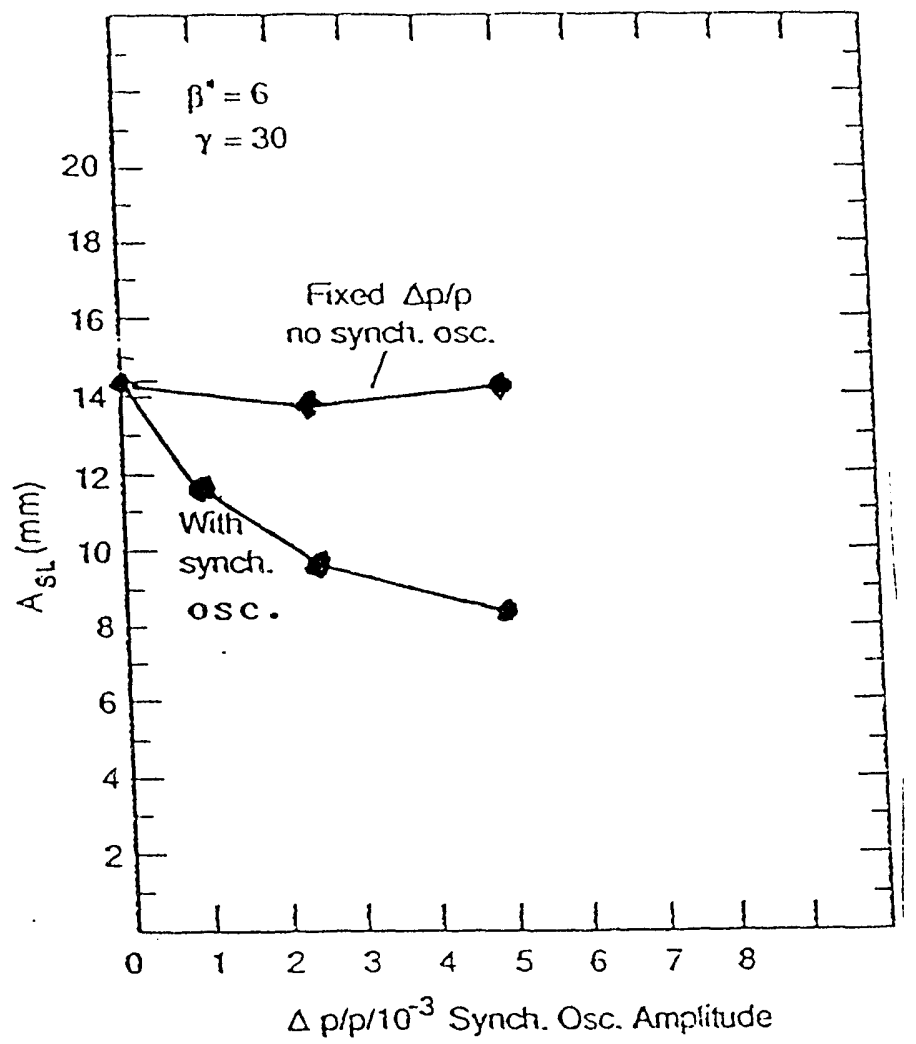

Figure 1: Comparison of results with and without synchrotron oscillations.

These tracking runs were done for 800,000 turns at $\gamma=30$ using the RHIC91 lattice with 6 insertions having $\beta^{*}=6 \mathrm{~m}$. One RF cavity is present when the synchrotron oscillations are included. All the results shown in Fig. 1 were found for the same distribution of field errors, which gave the smallest dynamic aperture of 10 distributions of errors studied at $\Delta p / p=0$.

The effect of the synchrotron oscillations on the dynamic aperture is studied further in Figs. 2 and 3. In Fig. 2, the survival time in turns is plotted against the initial betatron amplitude $x_{0}$ for four different synchrotron oscillation amplitudes, $\Delta p / p=0,0.001,0.0025$, and 0.005 , for a particular distribution of field errors which gave the smallest dynamic aperture of 10 distributions studied at $\Delta p / p=0$. The particle is started out with $p_{x 0}=p_{y 0}=0$ and $\epsilon_{x 0}=\epsilon_{y 0}$. The lattice is the same as was used for the results in Fig. 1. One sees that the survival curve moves towards lower $x_{0}$ as the synchrotron oscillation amplitude increases. Note that $x_{0}$ is the initial betatron oscillation, 


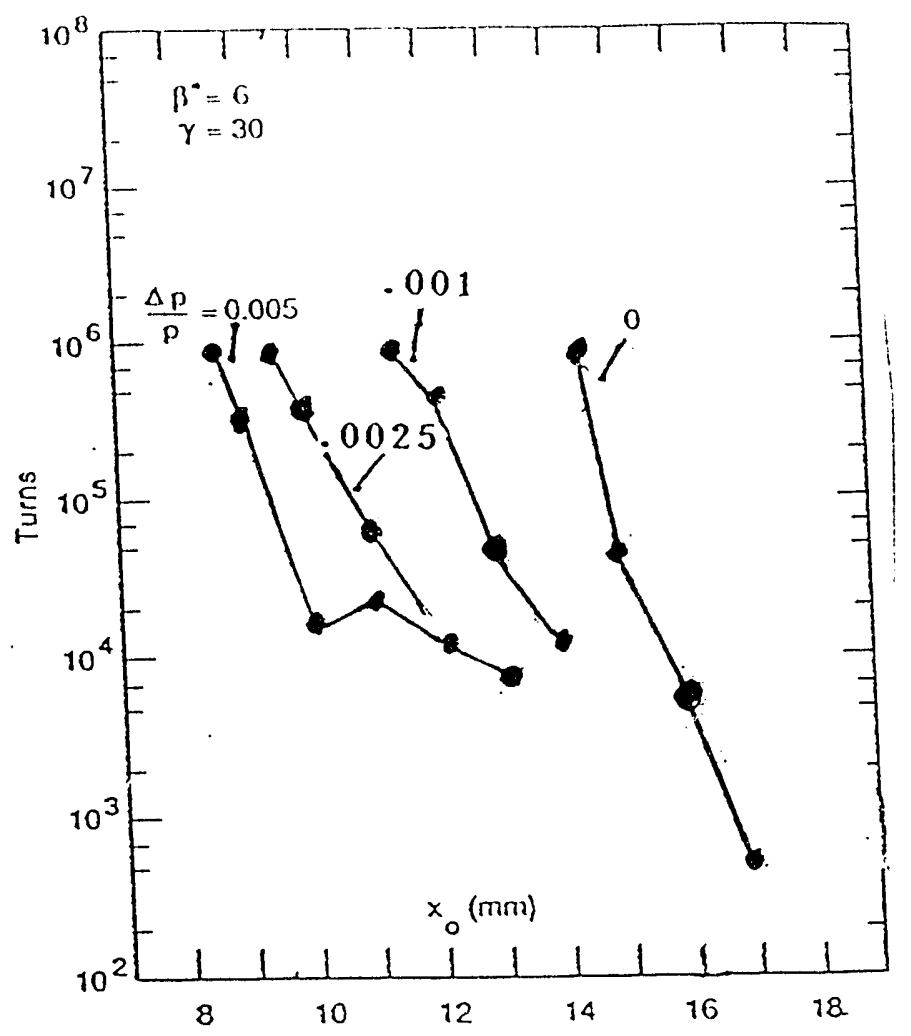

Figure 2: Comparison of results for different $\Delta p / p$.

defined as the initial $x$ minus the closed orbit position for the initial $\Delta p / p$, which is computed for a fixed $\Delta p / p$.

Fig. 3 compares survival plots, for this same worst distribution of field errors, for two cases. In one case, the RF is on and the particle has a certain synchrotron oscillation amplitude $\Delta p / p$. In the second case the RF is off, and the particle momentum is fixed. At $\Delta p / p=0$, the curves for the two cases do not differ greatly. For $\Delta p / p=0.005$, the two cases differ considerably; the $x_{0}$ to survive 800,000 turns is $x_{0}=8.5 \mathrm{~mm}$ for the case with the synchrotron oscillation amplitude $\Delta p=0.005$, compared to $x_{0}=14.5 \mathrm{~mm}$ for the case with no synchrotron oscillations and $\Delta p / p$ is fixed at $\Delta p / p=-0.005$.

\subsection{Comments on the Tracking}

It is important that the tracking be symplectic. To achieve this, the ORBIT program was changed to allow the use of point magnets. The methods used are similar to those used in the TEAPOT ${ }^{1}$ program, with some modifications. The RHIC91 lattice was used in the above studies. One $160 \mathrm{MHz}$ $\mathrm{RF}$ cavity with an RF voltage of $4.5 \mathrm{MV}$ was placed between $\mathrm{Q} 8$ and Q9 in one insertion region. The bucket height is $\Delta p / p=6 \times 10^{-3}$ at $\gamma=30$ and $\Delta p / p=2 \times 10^{-3}$ at $\gamma=100$. Random and systematic field errors were present 

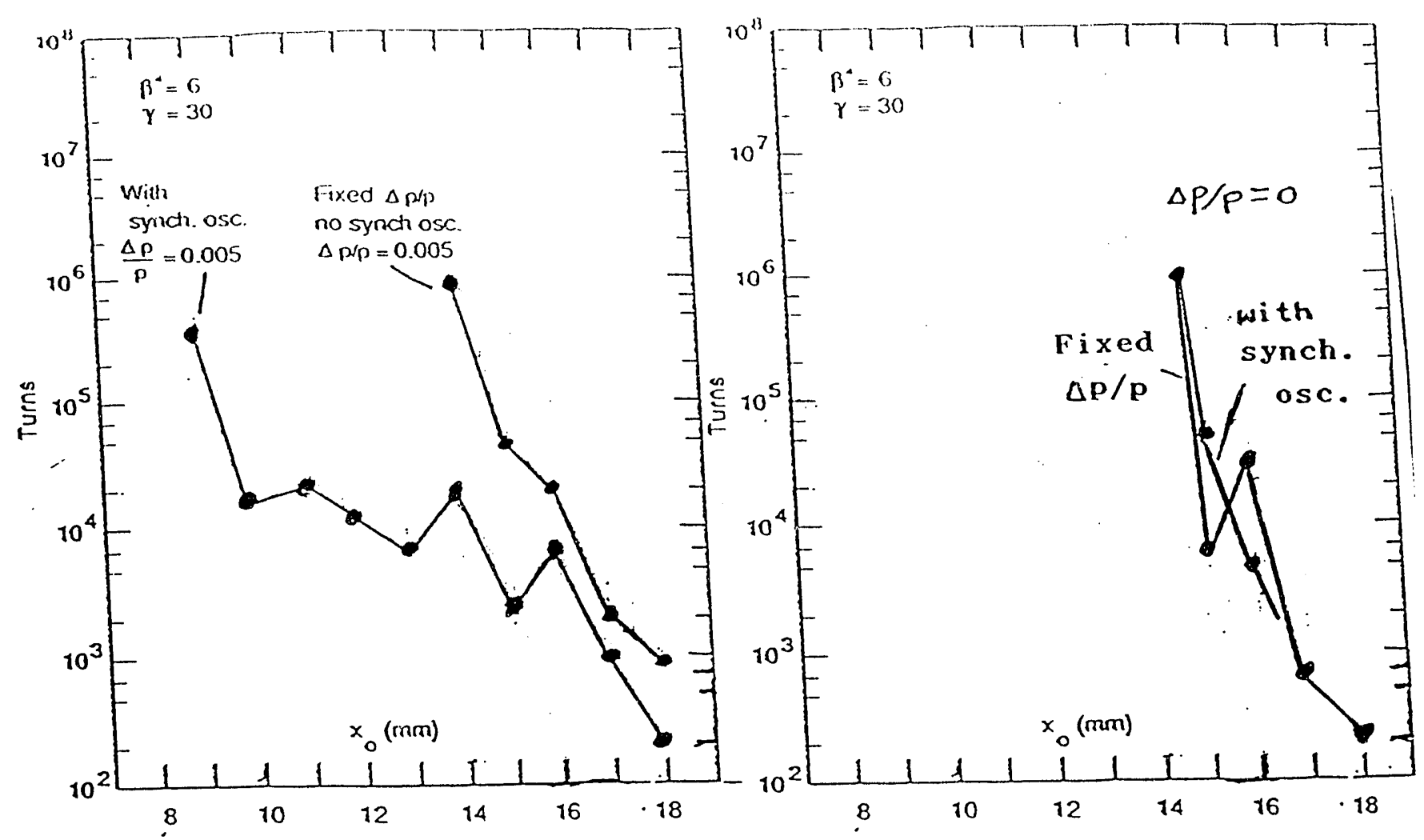

Figure 3: Comparison of results with and without synchrotron oscillations.

in each magnet at the level expected for RHIC. Field error multipoles up to order 10 were included. The nominal operating tune is $\nu_{x}=28.826, \nu_{y}=28.821$. To establish the dynamic aperture, the stability limit for 800,000 turns was examined for ten different distributions of the random field errors.

An interesting question is at what synchrotron oscillation amplitude, $\Delta p / p$, does the synchrobetatron coupling start to have an appreciable effect on the dynamic aperture, $A_{S L}$. The tracking studies indicate that it is the magnitude of $\Delta p / p$, rather than how close $\Delta p / p$ is to the edge of the RF bucket that is important. The tracking results suggest the following criterion for the $\Delta p / p$ where the synchrobetatron coupling becomes significant,

$$
X_{p} \frac{\Delta p}{p} \simeq A_{S L}
$$

where $X_{p}$ is the horizontal dispersion at the location in a normal cell where $A_{S L}$ is measured. $X_{p} \simeq 1.5 \mathrm{~m}$ in RHIC. Eq. (1) says that the synchrobetatron coupling becomes important when the particle transverse displacement due to $\Delta p / p$ is about equal to the betatron oscillation amplitude. The loss in dynamic aperture due to the presence of synchrotron oscillations appears to be roughly given by $X_{p} \Delta p / p$. 


\section{REFERENCE}

1. L. Schachinger and R. Talman, TEAPOT, A Thin Element Tracking Program, SSC-52 (1985). 

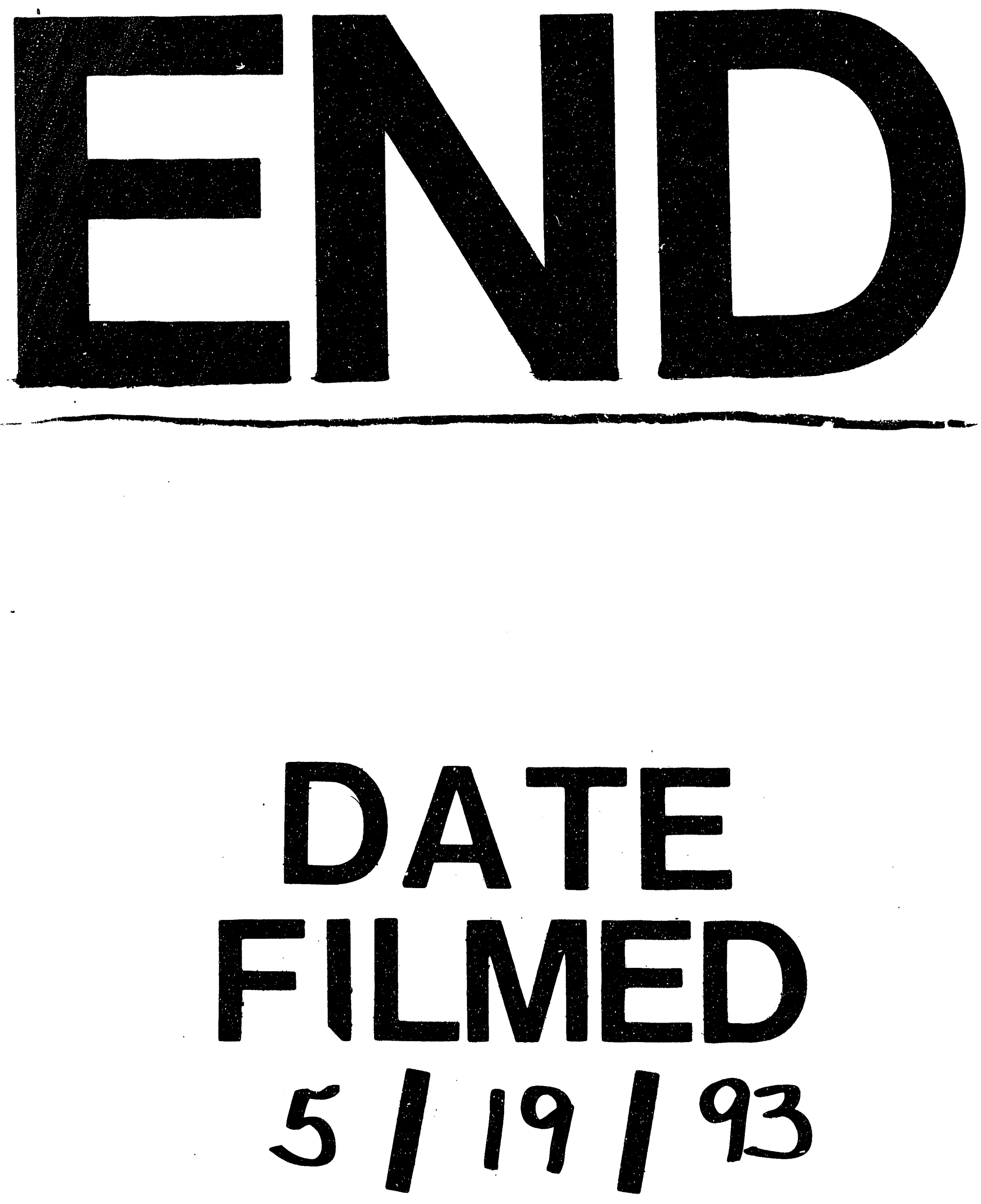
